\title{
GANANCIA GENÉTICA ESPERADA DE LA RESISTENCIA A SARNA COMÚN (Streptomyces spp.) EN UNA POBLACIÓN DE Solanum phureja Juz. et Buk.
}

\section{EXPECTED GENETIC ADVANCE FOR RESISTANCE TO COMMON SCAB IN A POPULATION FROM Solanum phureja Juz. et Buk.}

Catalina María Zuluaga Amaya • Elena Paola González Jaimes² • José Miguel Cotes Torres ${ }^{3}$

\section{RESUMEN}

La sarna común de la papa, causada por bacterias del género Streptomycetes, es una enfermedad de difícil manejo agronómico que se encuentra en todas las zonas de producción de papa en el mundo, por lo que la obtención de cultivares resistentes a la enfermedad es uno de los componentes del manejo integrado de la enfermedad. Hasta ahora se han obtenido algunos materiales resistentes en Estados unidos y Europa; no obstante los estudios genéticos realizados hasta ahora para determinar caracteres como la heredabilidad han dado pocos resultados que puedan ser utilizados para el mejoramiento genético. El presente estudio se realizó con la finalidad de estimar los parámetros genéticos de la resistencia a sarna común utilizando una población de 38 familias de medios hermanos de Solanum phureja en campo, en cuatro localidades diferentes del departamento de Antioquia (Colombia). Los resultados muestran que tres localidades fueron aptas para el análisis de los resultados por presentar diferentes niveles de severidad en los genotipos la otra localidad presentó un bajo nivel de severidad de la enfermedad lo que no permitió realizar una correcta selección. La heredabilidad en sentido estrecho osciló entre 0,22 y 0,45 para las localidades discriminantes. Se encontró una alta variación en la respuesta del genotipo al ambiente en la expresión de la resistencia genética. La ganancia genética esperada puede ser hasta del 20\% de disminución en severidad de la enfermedad por ciclo de selección, lo cual indica que el carácter de resistencia a sarna común puede ser manejado en programas convencionales de mejoramiento genético mediante esquemas de selección recurrente.

Palabras claves: Papa criolla, heredabilidad, Familias de hermanos medios, mejoramiento genético de papa

1 Agr. MSc. Profesor Asistente. Politécnico Colombiano Jaime Isaza Cadavid. Facultad de Ciencias Agrarias. Cr 48 No. 7-151 Medellín - Colombia. e-mail: catazuluaga81@gmail.com.

2 Agr. MSc. DSc. Profesor Asociado. Politécnico Colombiano Jaime Isaza Cadavid. Facultad de Ciencias Agrarias. Cr 48 No. 7-151 Medellín - Colombia. e-mail: epgonzalez@elpoli.edu.co.

3 Agr. MSc. DSc. Profesor Titular. Universidad Nacional Colombia - Sede Medellín - Facultad de Ciencias Agrarias - Departamento de Ciencias Agronómicas - Cll. 59A No. 63-20 - Núcleo El Volador, Medellín - Colombia. e-mail: jmcotes@unal.edu.co 


\begin{abstract}
The potato common scab caused by bacteria from genera Streptomycetes, is a disease with many difficulties to management in field conditions, widely distributed on the world. Thus, develop a new varieties with resistance to disease is an important advance in order to established an integrated management program to this disease. So far, varieties in United States and Europe were obtained, however there are a few information available about the heritability of the resistance and another genetic parameters that should be used for potato breeding programs. This research was carried out to study the parameter genetics of the resistance based on a population of 38 families from half-sib of Solanum phureja under field conditions. Four experiments in different sites were established in Antioquia (Colombia) in the harvest season of 2011. We found that three sites were useful to evaluate the genotype resistance to common scab. The heritability in narrow sense were between 0.22 and 0.45 , for this three useful sites. A high variation from genotypes within environments was founded. The expected genetic gain can be up to $20 \%$ decrease in disease severity by selection cycle, thus the trait of resistance to common scab can be improvement in conventional plant breeding programs using recurrent selection strategies.
\end{abstract}

Key words: Colombian creole potato, heritability, Half- sib families, Potato breeding.

\title{
INTRODUCCIÓN
}

La sarna común, es una enfermedad de importancia económica que afecta lo cultivos de papa a nivel mundial, la cual reduce la calidad de los tubérculos. La enfermedad ha sido reportada en América, India, Asia y África. La sarna también afecta otros cultivos de raíces y tubérculos como rábano, remolacha, zanahoria, nabo y papa dulce, donde puede generar considerables pérdidas económicas (Hill y Lazarovits, 2005). Este patógeno puede causar lesiones tipo superficial, eruptiva o profunda, siendo las lesiones eruptivas las más comunes. La mayoría de las lesiones tienen una apariencia elevada, áspera y corchosa. La infección de los tubérculos se da fundamentalmente a través de lenticelas inmaduras, por lo tanto son más susceptibles durante el período de rápido crecimiento de los tubérculos, que comienza cuando estos alcanzan un diámetro del doble de los estolones y continúa por aproximadamente seis a ocho semanas (Lorian et al., 1997).
La sarna común es causada por una bacteria del suelo tipo gram positiva del género Streptomyces spp. (Wanner, 2006). La especie más ampliamente descrita causando sarna común en papa es Streptomyces scabies (sinónimo Streptomyces scabiei), esta ha sido recientemente dividida en varias especies morfológicamente similares pero genéticamente diferentes (Bouchek-Mechiche et al., 2000a). Los Streptomycetes que causan sarna común en papa son clasificadas dentro de al menos 10 especies diferentes (Loria et al., 1997; Kreuze et al., 1999; Bouchek-Mechiche et al. 2000a; Park et al., 2003) y al menos tres especies adicionales causan "netted scab" en Europa o sarna reticulada en Norte América (Scholte y Labruyere 1985; Faucher et al., 1993; Loria et al., 1997; Bouchek-Mechiche et al. 2000b) y otras especies causan sarna en papa dulce (Clark et al., 1998). Sin embargo, son tres las especies causantes de sarna común de la papa más estudiadas 
a nivel mundial S.scabies, Streptomyces acidiscabies y Streptomyces turgidiscabies (Gouws, 2006). Según Bignell et al., (2010), estas tres especies de Streptomyces spp. no son específicas al huésped ni al tipo de tejido que atacan induciendo lesiones de sarna en diferentes tubérculos y raíces.

Por otro lado, Wanner y Haynes (2009) demostraron que los aislamientos de Streptomyces sp. difieren en agresividad de forma significativa. Estos autores, también observaron en ensayos realizados con cuatro diferentes cultivares de papa tratadas con aislamientos de Streptomyces spp. pertenecientes a diferentes especies y tipos moleculares, que existen interacciones específicas entre genotipo de plantaaislamiento del patógeno.

Los agricultores reportan que la sarna común es más severa y ocurre en cultivares de papa que previamente han sido considerados relativamente resistentes, las razones de esto han sido poco investigadas. La incidencia y severidad de la enfermedad varía en diferentes localidades y años, esto es debido en parte a la variación en las condiciones ambientales y la variación genética de los cultivares de papa (Wanner, 2006). Esto se confirma en trabajos realizados por Domkářová et al. (2007) y Sedláková et al. (2013) quienes encontraron diferencias significativas en resistencia a la sarna común entre variedades e híbridos, y entre años evaluados. Diferencias en la severidad de la sarna común sobre un mismo cultivar de papa creciendo en diferentes regiones también ha sido reportada por Haynes et al. (2006). Asimismo, Haynes et al. (2010) afirmaron que los resultados en las evaluaciones del germoplasma de papa pueden variar en severidad a la sarna común, por lo cual deben realizarse pruebas en múltiples ambientes para determinar la resistencia de cada material.

Los cambios en la incidencia entre los años puede ser el resultado de la cantidad del patógeno en el suelo como consecuencia de cambios relacionados con el clima que afectan la ecología del suelo, lo cual conduce a alterar las poblaciones patogénicas de Streptomycetes y/o sus competidores. Otra posibilidad es la aparición de nuevas especies causantes de sarna o aislamientos mejor adaptados a nuevas condiciones o cultivares de papa, o que han adquirido características de virulencia diferentes (Wanner, 2007).

Tegg y Wilson (2010), demostraron que la fitotoxina Taxtomina A es también uno de los factores que influencian la resistencia a la sarna común, además de otros factores anatómicos, químicos y fisiológicos del huésped. Cullen y Lees (2007) encontraron que también la virulencia del asilamiento afecta la respuesta de resistencia.

Como se ha demostrado, los cultivares de papa varían en su nivel de resistencia a la sarna. Sin embargo, hay muy pocos cultivares altamente resistentes y ninguno es inmune a la enfermedad. La resistencia a la sarna, es el objetivo de la mayoría de los programas de mejoramiento de la papa (Goth et al., 1993), sin embargo, existe desconocimiento acerca de la resistencia a la sarna, y de su herencia (Lorian et al., 1997). Haynes et al. (2009), afirman que la base genética de la resistencia a la sarna común, no ha sido satisfactoriamente elucidada.

Alam (1972), concluye que para la resistencia se requieren dos loci independientes, un alelo dominante en un locus y alelos homocigotos recesivos en el segundo locus. Murphy et al. (1995), transmitió resistencia a la sarna de diploides a tetraploides soportando así la hipótesis desarrollada por Alam. Sin embargo, estos estudios, no se diseñaron correctamente para determinar la herencia de la sarna común. La hipóstesis de uno o dos loci sugiere que la resistencia es heredable de una manera cuantitativa oligogénica, sin embargo, los resultados muestran que esta herencia puede ser de tipo cualitativa.

Hosaka et al. (2000), evaluaron la resistencia a sarna común en 100 accesiones de 18 especies silvestres de papa diploides durante tres ciclos y a partir 
de los cuales fueron seleccionadas 322 accesiones como resistentes; concluyeron que las especies ancestrales putativas de las papas cultivables (Solanum bukasovii, S. canasense y S. multidissectum) producen clones con alto grado de resistencia, debido a que estos son el origen de genes primarios que confieren esta resistencia.

Según Driscoll et al. (2009), la fenotipificación de la susceptibilidad a la sarna común es difícil por la gran variabilidad en los síntomas de la enfermedad entre los tubérculos de una misma planta, que varían desde la ausencia de sarna a presencia de hoyos profundos en los tubérculos. En trabajos realizados por y Haynes (2009), sugieren que mayor información sobre las interacciones planta-patógeno especificas combinado con el conocimiento de la distribución de las especies que causan sarna común, podrían constituir la base para recomendaciones exitosas de cultivares adecuados de papa, lo cual contribuirá a una fenotipificación más exacta y reproducible, necesaria para los estudios genéticos de resistencia a sarna común en la papa.

De Maine et al. (1993), reportaron que en las progenies diploides como tetraploides derivadas de parentales diploides resistentes el nivel de resistencia, fue menor en la progenie que en los parentales.

\section{Los agricultores reportan que la sarna común es más severa} y ocurre en variedades de papa que previamente han sido considerados relativamente resistentes, las razones de esto han sido poco investigadas.

los mismos autores, fue evaluada durante dos años sobre la reacción a la sarna en una población tetraploide, allí se observó una variación continua del fenotipo susceptible (cv. Jacqueline Lee) a la sarna común entre la progenie, con una distribución normal, lo que sugiere que el fenotipo de la enfermedad es una característica genéticamente compleja. También se observó una variación transgresiva, pero sesgada hacia la susceptibilidad. A su vez, en ensayos de invernadero fue posible distinguir individuos tolerantes y susceptibles. Existiendo una moderada correlación entre los estudios de invernadero y la prueba de campo para identificar clones resistentes a la sarna en una población segregante. Por su parte Wanner
Resultados similares se obtuvieron por Murphy et al. (1995), cuando diploides resistentes a la sarna con diferentes niveles de Solanum phureja Juz. et Buk., Soloanum stenotomum Juz. et Buk., y haploides de Solanum tuberosum subsp. tuberosum en su genealogía, fueron cruzados con el cultivar Shepody vía cruzamientos $4 x-2 x$, encontrando que en cinco de ocho familias el promedio de sarna fue menor en estas que los valores de los parentales. Así, ellos reportan una correlación moderadamente alta entre los índices de sarna de los parentales con sus familias, lo que implica que la información de resistencia de los parentales puede ser utilizada para determinar las combinaciones de los cruzamientos. 
Haynes et al. (2009), utilizando en campos naturalmente infestados por Streptomyces spp., realizaron estudios durante los años 2003-2005, para determinar la existencia de resistencia genética en una población diploide (271 clones) apareada al azar de S. phureja $\times$ S. stenotomum y estimaron la herencia de la resistencia en el sentido amplio $(H)$ y sentido restringido $\left(h^{2}\right)$ a la sarna común. Ellos hallaron diferencias significativas entre clones y la interacción años $x$ clon fue significativa. La $\mathrm{H}$ para la resistencia se estimó en 0,18 con un intervalo de confianza de 95\% entre 0,15 a 0,35. La fuente de variación clonal fue distribuida entre familias y dentro de familias. Toda la variación se presentó dentro de familias. No hubo variancia genética aditiva para resistencia en esta población, por consiguiente, $h^{2}$ fue estimado como 0.00 . En ese ensayo se pudo concluir que aunque los niveles de resistencia a la sarna común no pueden ser incrementados por métodos de selección en esta población diploide, puede ser posible transferir los niveles altos de resistencia de esta población al nivel tetraploide por medio de cruzamientos 4x-2x.

Más recientemente, en estudios realizados por Haynes et al. (2010), donde se evaluaron anualmente durante 2002 y 2007, entre 17-23 clones de papa en tres localidades, se determinó como respuestas en una distribución normal que hubo diferencias significativas entre clones para índice de área (IA) en dos años, índice de lesión (IL) en cinco años, y porcentaje de tubérculos con síntomas de sarna común (PS) en tres años. También, hubo interacciones significativas de clon x localidad para IA y PS en los seis años e IL en cinco años. La heredabilidad en sentido amplio para IA, IL, y PS fluctuó de 0 a 0,78, 0,49 a 0,90 y 0,30 a 0,80; respectivamente.

Como resultado de los diferentes programas de mejoramiento se han derivado algunas variedades de papa resistentes a sarna común para Europa y Estados Unidos como Western Russet (Love et al., 2006), GemStar Russet (Love et al., 2006), Kalaska
(Douches et al., 2009), Freedom Russet (Groza et al., 2009), Alta Crown (Bizimungu et al., 2011) y Teton Russet (Novy et al., 2014).

El objetivo de la presente investigación consistió en estimar la heredabilidad de la resistencia a la sarna común en una población de S. phureja en condiciones de campo, con el fin de favorecer el desarrollo de la selección de genotipos en el programa de Mejoramiento Genético de Papa Criolla que actualmente está desarrollando la Universidad Nacional de Colombia.

\section{MATERIALES Y MÉTODOS}

\section{Localización}

Para evaluar la resistencia a la sarna común se establecieron lotes de papa naturalmente infestados en el municipio de La Unión (Antioquia) veredas La Cabaña, Las Teresas, San Miguel y Vallejuelito, establecidos durante las siembras del 2011.

\section{Material vegetal}

Se tomaron 38 genotipos no emparentados de papa criolla con los cuales se construyó una población conformada por familias de medios hermanos, donde la semilla sexual de papa (TPS, True Potato Seed por sus siglas en inglés) se obtuvo a partir polinizaciones abiertas. La metodología para la extracción, lavado y almacenamiento de la semilla se hizo siguiendo las recomendaciones establecidas por el Centro Internacional de la Papa (CIP, 2010). A partir de TPS se obtuvo semilla prebásica de las 38 familias, de las cuales 29 familias tenían 20 hermanos medios y 9 familias entre 15 y 20 hermanos medios.

\section{Diseño experimental}

En campo se utilizó un diseño en Látice desbalanceado $7 \times 7$, con dos repeticiones; la unidad experimental fue constituida por familias con cinco hermanos medios y delimitada por plantas de la 
Para este modelo se asume que los vectores $f, h$, bye

variedad de papa Criolla Colombia como testigo susceptible a la enfermedad. La distancia entre surcos fue de $1,0 \mathrm{~m}$ y 0,3 $\mathrm{m}$ entre plantas.

Al momento de la cosecha los tubérculos fueron lavados con agua corriente para realizar la evaluación visual de los síntomas de sarna común, utilizando la escala diagramática la cual cuantifica el porcentaje de tejido afectado en la superficie del tubérculo, dividida en seis categorías de cero a cinco, siendo cero la ausencia de síntomas visuales y las demás categorías divididas así: $1=$ entre $0 \%$ y $5 \% ; 2=$ entre 5 y $10 \% ; 3=$ entre 0 y $25 \%$; $4=$ entre 25 y $50 \%, 5=$ mayor del $50 \%$, teniendo en cuenta que los límites inferiores de clase son abiertos, es decir que el valor exacto nunca es alcanzado, así por ejemplo en la categorías dos son todos aquellos porcentajes mayores que $5 \%$, sin incluir el valor exacto del $5 \%$.

\section{Análisis estadístico}

Para el análisis estadístico se consideró la variable severidad de la enfermedad para lo cual se tomó el valor de la marca de clase de las seis categorías de la escala diagramática utilizada (Madden et al., 2007).

En cada localidad, para la obtención de los componentes de varianza que permiten calcular la heredabilidad de la característica de resistencia a Streptomyces spp., se utilizó un modelo genético aditivo lineal univariado (Sorensen y Gianola, 2002). El modelo asumido fue el siguiente:

\section{$\mathbf{y}=\mathbf{1} \mu+\mathbf{z}_{1} \mathbf{f}+\mathbf{z}_{2} \mathbf{h}+\mathbf{z}_{3} \mathbf{b}+\mathbf{e}$}

donde: $y$ es el vector de observaciones de tamaño $n$ de la variable incidencia de la enfermedad. $\mu$ es la media general de la incidencia de la enfermedad; $f$ es el vector de efectos genéticos de las familias; $h$ es el vector de efectos genéticos de hermanos medios $b$ es un vector de efectos ambientales asociado a los bloques completos. Las matrices de incidencia $Z$ localizan el valor de los efectos correspondiente a cada dato observado. se distribuyen normal con media cero y varianzas $\mathbf{I} \sigma_{f}^{2} \mathbf{I} \sigma_{h}^{2}$ , $\mathbf{I} \sigma_{\mathrm{b}}^{2}$ y $\mathbf{I} \sigma_{\mathrm{e}}^{2}$. Para la estimación de los parámetros se utilizó la metodología de estimación Bayesiana, obteniendo como estimativa la mediana de la distribución a posteriori, que es aquel valor que minimiza el riego de bayes bajo la función de pérdida absoluta. Para todos los parámetros se utilizaron distribuciones a priori no informativas (Sorensen y Gianola, 2002). Para el análisis de los datos se utilizó el programa R (R Development Core Team, 2012) con el paquete MCMCglmm (Hadfield, 2010), el cual implementa el algoritmo de GIBBS para ser usado en este tipo de modelos. Se obtuvo una cadena de Markov de 1.030.000 de la distribución a posteriori conjunta de cada parámetro, considerando las primeras 10.000 iteraciones como período de burn-in, y para la obtención de los valores de las distribuciones marginales de cada parámetro se consideró tomar una muestra de cada 10 generadas.

Asimismo, con base en los valores obtenidos en la cadena de Markov, se estimó la heredabilidad de la característica. La heredabilidad depende de la población en estudio y de la unidad de selección que se utilice, calculándose como el cociente entre la varianza aditiva entre unidades de selección y la varianza fenotípica entre unidades de selección (Cruz y Carneiro, 2003). Así, la heredabilidad se evaluó bajo los métodos de selección entre familias, entre y dentro de familias, masal estratificada y masal simple (Tabla 1)

Se calculó la ganancia genética esperada para cada método de mejoramiento mediante la expresión:

$$
\text { Ganancia Esperada }=\mathbf{i} \times \mathrm{h}^{2} \times \hat{\sigma}_{\mathrm{p}}
$$

donde $i$ es la intensidad de selección y depende de la proporción de la población a ser parte del grupo de los genotipos seleccionados y de los valores fenotípicos correspondientes a una distribución normal, $h^{2}$ es la heredabilidad en sentido estrecho dependiendo del método de selección y $\hat{\sigma}_{\mathrm{p}}$ es la desviación estándar fenotípica por unidad de selección (Falconer y Mackay, 1996). 
Tabla 1. Estimación de las varianzas aditiva y fenotípica por unidad de selección, dependiendo del método de mejoramiento.

\begin{tabular}{ccc}
\hline Método de Selección & $\begin{array}{c}\text { Varianza aditiva por unidad } \\
\text { de selección }\end{array}$ & $\begin{array}{c}\text { Varianza fenotípica por } \\
\text { unidad de selección }\end{array}$ \\
\hline Entre familias & $\hat{\sigma}_{f}^{2}$ & $\hat{\sigma}_{\mathrm{f}}^{2}+\frac{1}{2} \hat{\sigma}_{\mathrm{h}}^{2}+\frac{1}{10} \hat{\sigma}_{\mathrm{e}}^{2}$ \\
\hline Entre y dentro de familias & $3 \hat{\sigma}_{\mathrm{f}}^{2}$ & $\hat{\sigma}_{\mathrm{e}}^{2}$ \\
\hline Masal estratificada & $4 \hat{\sigma}_{\mathrm{f}}^{2}$ & $\sigma_{\mathrm{f}}^{<}+\sigma_{\mathrm{h}}^{<}+\sigma_{\mathrm{e}}^{<}$ \\
\hline Masal simple & $4 \hat{\sigma}_{\mathrm{f}}^{2}$ & $\hat{\sigma}_{\mathrm{b}}^{2}+\hat{\sigma}_{\mathrm{f}}^{2}+\hat{\sigma}_{\mathrm{h}}^{2}+\hat{\sigma}_{\mathrm{e}}^{2}$ \\
\hline
\end{tabular}

\section{RESULTADOS Y DISCUSIÓN}

En la evaluación de severidad cada localidad mostró un comportamiento diferente respecto a la enfermedad, tanto en magnitud como en el ranking de las familias en las diferentes localidades. Es así como, La Cabaña, Las Teresas y Vallejuelito presentaron un mayor nivel de enfermedad (Figura 1). El testigo en estas tres veredas presentó valores superiores a $25 \%$ de severidad del tubérculo.

En el caso de San Miguel donde el nivel de enfermedad fue menor que en las demás localidades, no fue posible diferenciar las familias por su grado de resistencia ya que todas presentaron una baja severidad de la enfermedad, lo cual indica que esta localidad no permite hacer una selección por resistencia a sarna común (Figura 1C). Además en ésta localidad el testigo presentó un valor inferior a $10 \%$ de severidad en tubérculos, corroborando así el menor desarrollo de la enfermedad en esta localidad. Dentro de las localidades evaluadas, Las Teresas y Vallejuelito son las localidades que permiten discriminar de mejor forma la respuesta de las familias por su resistencia a la sarna común, ya que se observa mayor variabilidad y una mejor precisión del valor estimado de la severidad, siendo los intervalos de alta densidad a posteriori más cortos que en las otras localidades.
En Las Teresas, la familia 40 presenta un valor de severidad de $15 \%$, siendo el mayor valor reportado comparado con las demás familias en todas las localidades. La familia 2 se encontró como susceptible en las cuatro localidades evaluadas en el departamento de Antioquia, lo que permite clasificar a esta familia como la más susceptible con un valor promedio de $10 \%$ de severidad (Figura 1). Por otro lado, las familias 57 y 67 son en términos generales las más resistente al patógeno con un valor promedio de $6 \%$, lo cual indica que dentro del acervo genético de estas familias no existen genes que confieran resistencia completa al patógeno.

En la tabla 2 se observa que, en general, existe una baja correlación Pearson-Spearman en las localidades, indicando una fuerte variación en la respuesta del genotipo a los ambientes evaluados. Encontrando inclusive correlaciones negativas de Spearman y Pearson entre las localidades La Cabaña y Vallejuelito; y con un máximo valor de correlación del 0,3034 para Spearman entre San Miguel y Vallejuelito; y 0,3382 para Pearson entre La Cabaña y San Miguel; los cuales son bajos. No obstante cabe resaltar que estas dos localidades tuvieron una respuesta contrastante respecto a su capacidad de discriminación de los genotipos como se observa en la Figura 1. Estos hallazgos confirman lo reportado por Wanner (2006); Haynes et al. (2006); Domkářová 

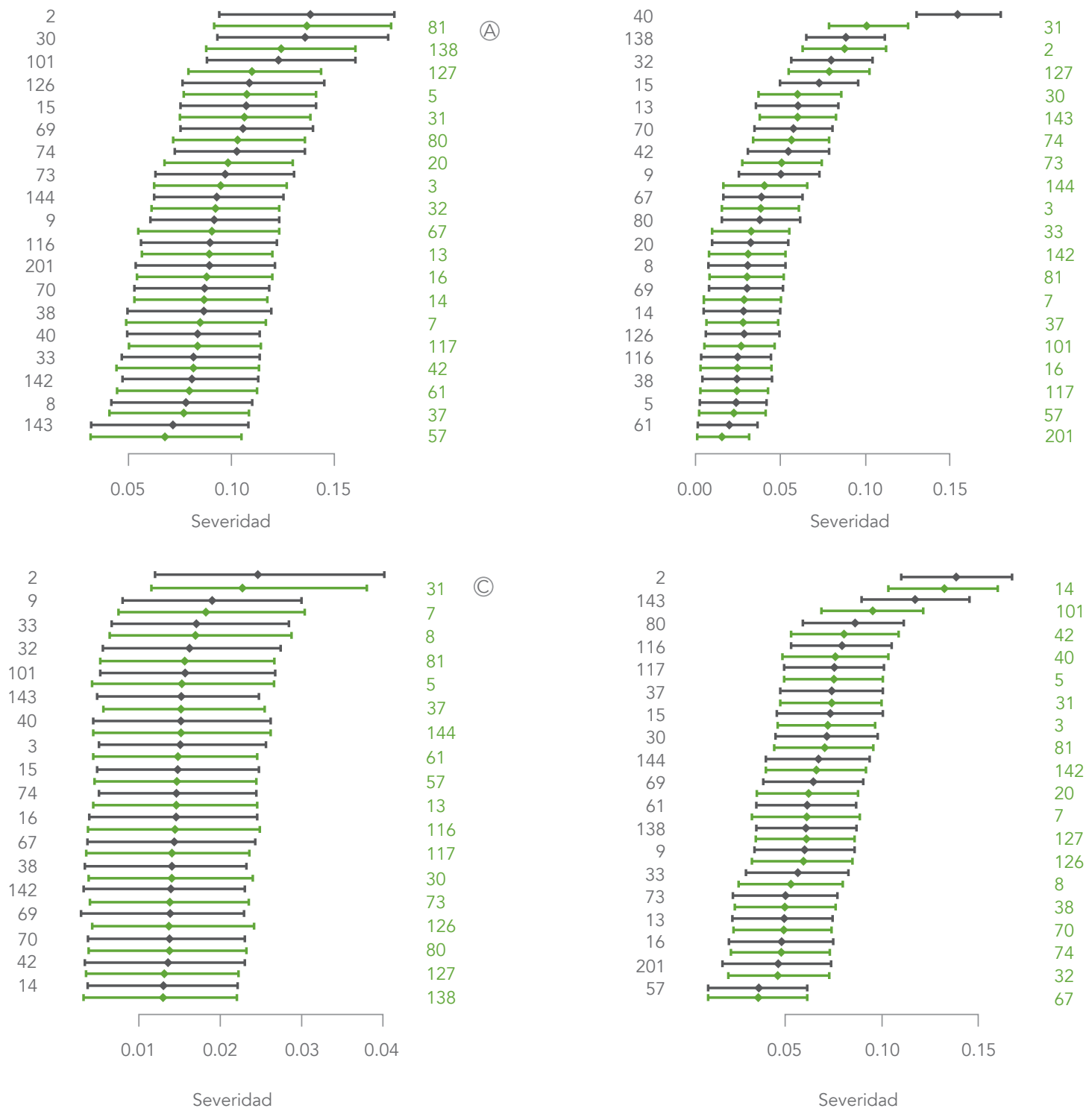

Figura 1. Predictores lineales bayesianos (BLP) para el efecto de 38 familias maternas para la severidad de sarna común en tubérculos en cuatro localidades del municipio de La Unión. Veredas A) La Cabaña; B) Las Teresas; C) San Miguel y D) Vallejuelito. Las barras de error indican los límites del intervalo de alta densidad a posteriori de $90 \%$.

et al. (2007) y Sedláková et al. (2013); quienes afirman que la incidencia y severidad de la enfermedad varía entre localidades y temporadas, debido en parte a la variación en las condiciones ambientales y la variación genética de los cultivares de papa. Además, de la importancia de realizar evaluaciones del germoplasma de papa para determinar la resistencia de cada material en diferentes localidades como lo sugieren Haynes et al. (2010). 
Tabla 2. Correlación para la severidad de la enfermedad en diferentes localidades. El asterisco indica diferencias significativas $(\boldsymbol{\alpha}=0.05)$ para la $H_{0}: \rho$ $=0$. En la matriz triangular superior se presenta el coeficiente de correlación de Spearman y en la inferior el coeficiente de correlación lineal de Pearson.

\begin{tabular}{ccccc}
\hline & La Cabaña & Las Teresas & San Miguel & Vallejuelito \\
\hline La Cabaña & 1 & 0,0938 & $0,2951^{\star}$ & $-0,1916^{\star}$ \\
\hline Las Teresas & $0,1812^{\star}$ & 1 & $0,1507^{\star}$ & $0,1404^{\star}$ \\
\hline San Miguel & $0,3382^{\star}$ & $0,2326^{\star}$ & 1 & $0,3034^{\star}$ \\
\hline Vallejuelito & $-0,192^{\star}$ & $0,2661^{\star}$ & $0,2316^{\star}$ & 1 \\
\hline
\end{tabular}

La mayor heredabilidad se obtuvo en la vereda Las Teresas, bajo cualquiera de los sistemas de selección evaluados, seguido de los obtenidos en la vereda Vallejuelito, obteniendo en ambas localidades valores superiores a los obtenidos por Haynes et al. (2009) y Haynes et al. (2010), mostrando así que esta fuente de germoplasma puede ser utilizada para obtener poblaciones con mayores niveles de resistencia a la enfermedad (Tabla 3). Se confirma que en la vereda de San Miguel la variación de severidad de la enfermedad entre las familias y entre los individuos en muy poca, obteniéndose valores de parámetros genéticos marginales, menores a 0,0001.

Tabla 3. Parámetros genéticos estimados para la severidad en tubérculos de la sarna común en cuatro localidades del municipio de La Unión (Antioquia).

\begin{tabular}{cccccc}
\hline Parámetro & La Cabaña & Las Teresas & San Miguel & Vallejuelito & General \\
\hline$\sigma_{\text {Bloque }}^{2}$ & $<0,0001$ & $<0,0001$ & $<0,0001$ & $<0,0001$ & $<0,0001$ \\
\hline$\sigma_{\text {Familia }}^{2}$ & 0,0007 & 0,0010 & $<0,0001$ & 0,0008 & 0,0004 \\
\hline$\sigma_{\text {HS(Familia) }}^{2}$ & 0,0002 & 0,0000 & $<0,0001$ & $<0,0001$ & $<0,0001$ \\
\hline$\sigma_{\text {Error }}^{2}$ & 0,0108 & 0,0045 & $<0,0001$ & 0,0057 & 0,0165 \\
\hline$h_{\text {Familiar }}^{2}$ & 0,5037 & 0,8125 & $<0,0001$ & 0,7213 & 0,2988 \\
\hline$h_{\text {Familiar /Individual }}^{2}$ & 0,1827 & 0,6536 & $<0,0001$ & 0,3913 & 0,0674 \\
\hline$h_{\text {MasalEstratificada }}^{2}$ & 0,2240 & 0,7153 & $<0,0001$ & 0,4612 & 0,0873 \\
\hline$h_{\text {Masal }}^{2}$ & 0,2207 & 0,7120 & $<0,0001$ & 0,4535 & 0,0826 \\
\hline
\end{tabular}

No se presentan diferencias estadísticamente significativas entre las ganancias genéticas esperadas para los cuatro métodos de selección evaluados en las localidades evaluadas. En la localidad de San Miguel, (Figura 2), la ganancia genética esperada tiende a cero, aun cuando se hace una presión de selección del 20\%. En las demás localidades si se presentan ganancias genéticas esperadas para los cuatro métodos de selección, obteniéndose mayores estimaciones puntuales cuando se utiliza el método de selección masal o individual, con un valor máximo de ganancia de $9 \%$ de severidad cuando se aplica una presión de selección baja como se observa en Las Teresas. 
Teniendo en cuenta que en las mejores localidades, los intervalos de alta densidad a posteriori, la ganancia esperada puede llegar a ser del 20\% de severidad de la enfermedad por ciclo de selección, podemos concluir que el carácter de resistencia a

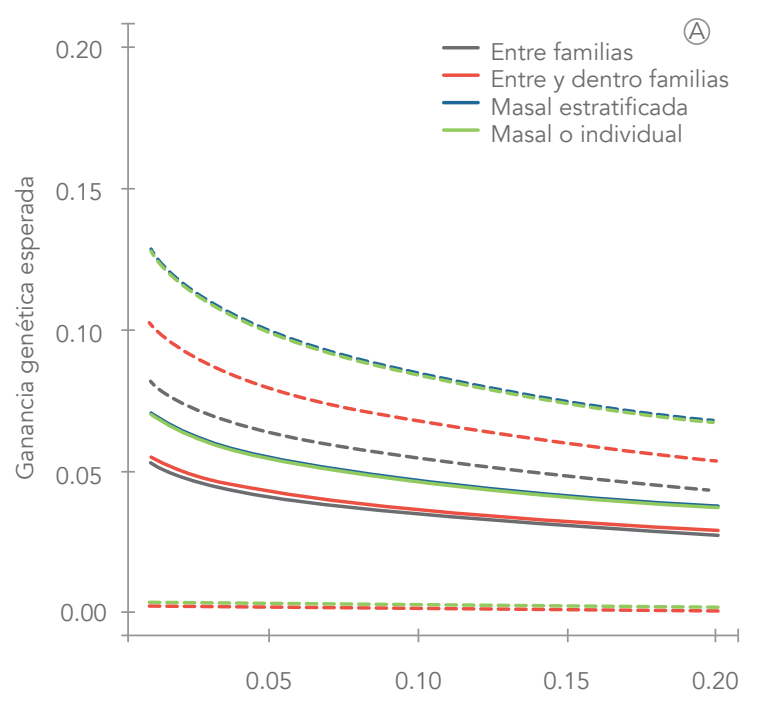

Proporción de selección

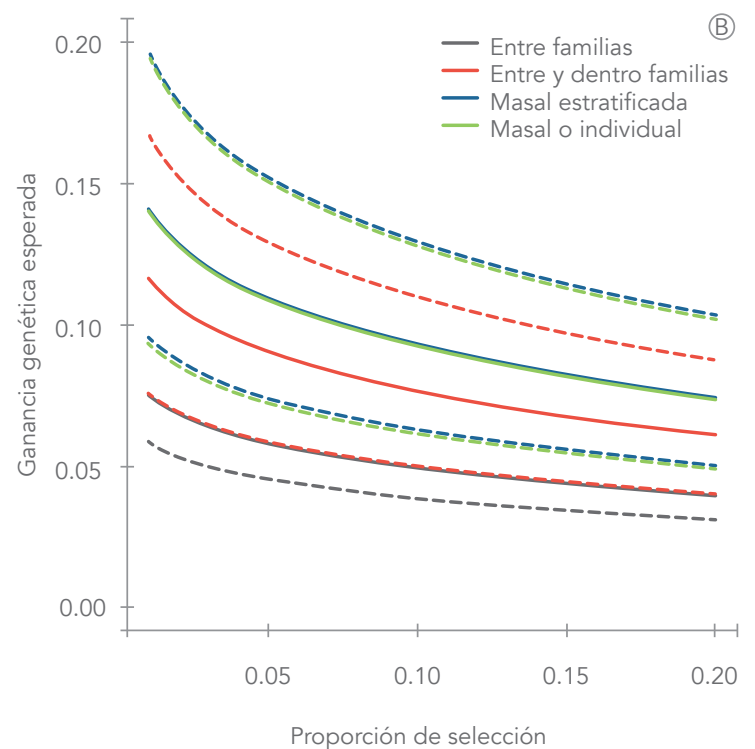

sarna común responde a métodos convencionales de mejoramiento genético de plantas, y un esquema de selección recurrente sería apropiado para mejorar la resistencia a sarna común en la población de $S$. phureja utilizada en el estudio.
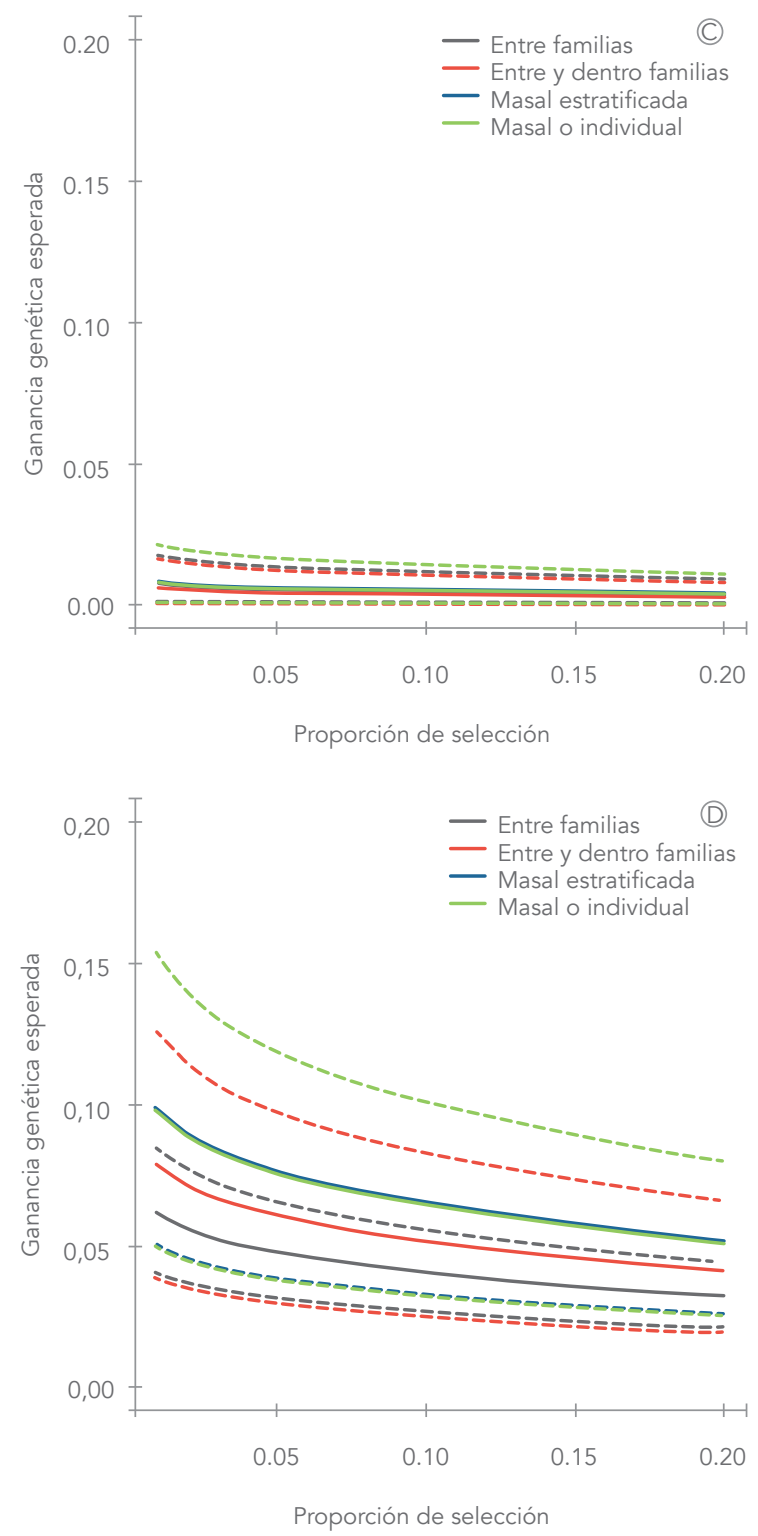

Figura 2. Ganancias genéticas esperadas en la población de $S$. phureja para la severidad de la sarna común en tubérculos para diferentes métodos de selección en cuatro localidades del municipio de La Unión (Antioquia). Veredas A) La Cabaña; B) Las Teresas; C) San Miguel y D) Vallejuelito. Las líneas sólidas representan el valor estimado de Bayes y las líneas discontinuas los límites del intervalo de alta densidad a posteriori de $90 \%$. 


\section{REFERENCIAS}

1. Bizimungu B., Holm D.G., Kawchuk L.M., Konschuh M., Schaupmeyer C., Wahab J., Waterer D., Driedger D., Wolfe H., McAllister P., Howard R., Platt H. W. y Lynch D.R. 2011. Alta Crown: A New Russet Potato Cultivar with Resistance to Common Scab and A Low Incidence of Tubers Deformities. American Journal of Potato Reserach 88:72-81.

2. Bignell D.R., Hunguet-Tapia J.C., Joshi M.V., Pettis G.S. y Loria R. 2010. What does it take to be a plant pathogen: genomic insights from Streptomyces species. Antonie van Leeuwenhoek 98: 179-94.

3. Bouchek-Mechiche K., Gardan L., Normand P., y Jouan B. 2000a. DNA relatedness among strains of Streptomyces pathogenic to potato in France: Description of three new species, S. europaeiscabiei sp. nov. and S. stelliscabiei sp. nov. associated with common scab, and S. reticuliscabiei sp. nov. associated with netted scab. International Journal of Systematic and Evolutionary Microbiology 50:91-99.

4. Bouchek-Mechiche K., Pasco C., Andrivon D., y Jouan B. 2000b. Differences in host range, pathogenicity to potato cultivars and response to soil temperature among Streptomyces species causing common and netted scab in France. Plant Pathology 49:3-10.

5. CIP 2010. Procedimientos para pruebas de evaluación estándar de clones avanzados de papa. Lima, Perú, 152 p.

6. Clark C.A., Chen A., Ward-Rainey N., y Pettis G.S. 1998. Diversity within Streptomyces ipomoeae based on inhibitory interactions, REPPCR, and plasmid profiles. Phytopathology 88:1179-1186.

7. Cruz C.D.; Carneiro P.C. 2003. Modelos biométricos aplicados ao melhoramento genético Vol 2. Viçosa, Universidade Federal de Viçosa (UFV). 585 p.

8. Cullen D.W. y Lees A.K. 2007. Detection of the nec1 virulence gene and its correlation with pathogenicity in Streptomyces species on potato tubers and in soil using conventional and real-time PCR. J. Appl. Microbiol. 102: 1082-1094.

9. Domkárová J., Vokál B., Horácková V. y Brož J. 2007. The greenhouse provocation test for determination of resistance to potato common scab [Streptomyces scabiei (ex Thaxter 1982) 
Lambert and Loria 1989]. Plant soil environment 53: 72-80.

10. Douches D.S., Coombs J., Hammerschmidt R., Kirk W.W. y Long, C. 2009. Kalkaska: A Round White Chip-Processing Potato Variety with Common Scab Resistance. American Journal of Potato Research 86:347-355.

11. Driscoll J., Coombs J, Hammerschmidt R, Kirk W., Wanner L. y Douches D. 2009. Greenhouse and Field Nursery Evaluation for Potato Common Scab Tolerance in a Tetraploid Population. American Journal of Potato Research 86:96-101.

12. Falconer D.S. y Macray T. 1996. Introduction to quantitative genetics. 4th edition. England: Longman. 464 p.

13. Faucher E., Otrysko B., Paradis E., Hodge N.C., Stall R.E. y Beaulieu C. 1993. Characterization of streptomycetes causing Russett scab in Quebec. Plant Disease 77:1217-1220.

14. Goth R.W., Haynes K.G. y Wilson, D.R. 1993. Evaluation and characterization of advanced potato breeding clones for resistance to scab by cluster analysis. Plant Disease 77:911-914.

15. Gouws R. 2006. Etiology and integrated control of common scab on seed potatoes in South Africa. Master's Thesis. University of Pretoria, South Africa. 128p.

16. Groza H.I., Bowen B.D., Bussan A.J., Navarro F.M., Stevenson W.R., Palta J. P. y Jiang J. 2009. Freedom Russet-A Dual Purpose Russet Potato Cultivar with Resistance to Common Scab and Good Fry Quality. American Journal of Potato Research 86:406-414.

17. Hadfield J.D. 2010. MCMC Methods for MultiResponse Generalized Linear Mixed Models: The MCMCglmm R Package. Journal of Statistical Software 33: 1-22.

18. Haynes K.G, Wanner L.A., Thill C.A., Novy R.G. y Whitworth J. L. 2006. Common scab trials of potato varieties and advanced selections in 2003. American Journal of Potato Research 83:113

19. Haynes K.G., Christ B.J., Burkhart C.R. y Vinyard B. T. 2009. Heritability of Resistance to Common Scab in Diploid Potatoes. American Journal of Potato Research 86:165-170.

20. Haynes K. G., Wanner L.A., Thill C.A., Bradeen J.M., Jeffrey M., Novy R.G., Whitworth J.L., Corsini, D.L. y Vinyard B.T. 2010. Common 
Scab Trials of Potato Varieties and Advanced Selections at Three U.S. Locations. American Journal of Potato Research 87:261-276.

21. Hill J. y Lazarovits G. 2005. A mail survey of growers to estimate potato common scab prevalence and economic loss in Canada. Canadian Journal of Plant Pathology 27:46-52.

22. Hosaka K., Matsunaga H. y Senda K. 2000. Evaluation of Several Vtrfld Tuber-bearing Solanum Species for Scab Resistance. American Journal of Potato Research 77:41-45.

23. Kreuze J.F., Suomalainen S., Paulin L. y Valkonen, J.P.T. 1999. Phylogenetic analysis of $16 \mathrm{~S}$ rRNA genes and PCR analysis of the nec1 gene from Streptomyces spp. Causing common scab, pitted scab, and netted scab in Finland. Phytopathology 89:462-469.

24. Loria R., Bukhalid R.A., Fry B.A. y King R.R. 1997. Plant pathogenicity in the genus Streptomyces. Plant Disease 81:836-846.

25. Love S.L., Novy R.G., Whitworth J., Corsini, D.L., Pavek J. J., Mosley A.R., Pavek M.J., Knowles N.R., Brown C.R., James S. R. y Hane D. C. 2006. Western Russet: A New Potato Variety with Excellent Fresh Market and
Frozen-fried Processing Quality and Field Resistance to Common Scab and PVY. American Journal of Potato Research 83:161-169

26. Love S. L., Novy R. G., Whitworth J., Corsini D.L., Pavek J.J., Mosley A.R., Pavek M.J., Knowles N.R., Brown C.R., James S.R., Hane D.C. y Miller J.C. 2006. GemStar Russet: A Potato Variety with High Yield, Good Culinary Quality, Excellent Fresh Market Appearance, and Resistance to Common Scab. American Journal of Potato Research 83:171-180

27. Madden, L. V., Hughes, G. y van den Bosch F. 2007. The Study of Plant Disease Epidemics. APS Press, St. Paul, MN. 432 p.

28. Merz U. 2000. Powdery scab. Research in Switzerland. Past and present Research: Powdery SCAF. p. 67-71. En: Merz U. y Lees A.K. (Eds.) Proceedings of the First European Powdery scab Workshop July 20-22. Scottish Crop Research Institute Aberdeen, Scottland.110p.

29. Novy R.G., Whitworth J.L., Stark J.C., Charlton B.A., Yilma S., Knowles N.R., Pavek M. J., Spear R.R., Brandt T.L., Olsen N., Thornton M. y Brown, C.R. 2014. Teton Russet: An Early-Maturing, Dual-Purpose Potato Cultivar Having 
Higher Protein and Vitamin C Content, Low Asparagine, and Resistances to Common Scab and Fusarium Dry Rot. American Journal of Potato Research 91:380-393.

30. Park D.H., Kim J.S., Kwon S.W., Wilson C., Yu Y.M., Hur J.H. y Lim C.K. 2003. Streptomyces luridiscabiei sp. nov., Streptomyces puniciscabiei sp. nov. and Streptomyces niveiscabiei sp. nov., which cause potato common scab disease in Korea. International Journal of Systematic and Evolutionary Microbiology 53:2049-2054.

31. R Development Core Team. 2012. R: A Language and Environment for Statistical Computing. R Foundation for Statistical Computing. Vienna, Austria. http://www.R-project.org/

32. Scholte K. y Labruyere R.E. 1985. Netted scab: A new name for an old disease in Europe. Potato Research 28:443-448.

33. Sedláková V., Dejmalová J. , Dolezal P., Hausvater E., Sedlák P., Bastová P. 2013. Characterization of forty-four potato varieties for resistance to common scab, black scurf and silver scurf. Crop Protection 48: 82-87.

34. Soomro Z.A., Kumbhar A.S., Larik, A.S., Imram, M. y Brohi, S.A. 2010. Heritability and selection response in segregating generations of upland cotton. Pakistan Journal of Agricultural Research 23: 25-30.

35. Sorensen D. y Gianola, D. 2002. Likelihood, Bayesian, and MCMC Methods in Quantitative Genetics. First Edition. Springer-Verlag, New York. 740p.

36. Tegg R.S. y Wilson C.R. 2010. Relationship of resistance to common scab disease and tolerance to thaxtomin A toxicity within potato cultivars. European Journal of Plant Pathology 128:143-148.

37. Wanner L.A. 2006. A Survey of Genetic Variation in Streptomyces Isolates Causing Potato Common Scab in the United States. Bacteriology. 96: 1363-1371.

38. Wanner L.A. 2007. A New Strain of Streptomyces Causing Common Scab in Potato. Plant Disease. 91: 352-359.

39. Wanner L.A. y Haynes K. G. 2009. Aggressiveness of Streptomyces on Four Potato Cultivars and Implications for Common Scab Resistance Breeding. American Journal of Potato Research 86:335-346. 